\title{
Estudio palinológico de un perfil sedimentario del Holoceno medio-tardío, oeste del Iberá, Corrientes, Argentina
}

\section{Lionel Fernandez Pacella}

Universidad Nacional del Nordeste, Centro de Ecología Aplicada del Litoral, (CECOAL-CONICET-UNNE), CP 3400-Corrientes, Argentina.

lionelpacella@yahoo.com.ar

\section{RESUMEN}

Con la denominación "Iberá", identificamos a uno de los humedales tropicales más importantes de la biósfera en términos de su extensión $\mathrm{y}$ de las especies que lo habitan, tanto animales como vegetales. El objetivo de este trabajo es a partir del análisis polínico y litológico de un perfil sedimentario, reconstruir las comunidades vegetales predominantes y sus variaciones, inferir los cambios en las condiciones climáticas que se habrían producido y deducir el ambiente deposicional durante el Holoceno medio y tardío en la región del Iberá. El perfil fue muestreado sobre una barranca ubicada en la zona central de la margen occidental del Sistema Iberá, en la localidad de San Miguel. La dinámica de la vegetación y la relación de los taxones permitieron establecer tres zonas polínicas: La Zona Polínica I (Holoceno medio) presenta una asociación característica de la estepa graminosa. Las litofacies identificadas sugieren un ambiente depositacional de valles anegadizos, que junto a las paleocomunidades vegetales reconocidas, señalarían para el Holoceno medio, condiciones húmedas. Las Zonas Polínicas II y III (Holoceno tardío) registran una asociación característica de estepa herbácea psamófila. El aumento observado en las Brassicaceae, estaría indicando disturbios ambientales, los cuales podrían reflejar la combinación de una intensa acción eólica bajo un clima árido. Las litofacies identificadas señalan un ambiente eólico con derrames fluviales y junto a las comunidades vegetales reconocidas, indicarían para el Holoceno tardío, condiciones áridas a semiáridas.

Palabras clave: palinología; Holoceno; Iberá; reconstrucción; ambiente; clima; Argentina.

\begin{abstract}
With the denomination "Iberá", we identify one of the most important tropical wetlands of the biosphere in terms of its extension and living species, both animal and vegetal. The objective of this paper is to reconstruct the predominant plant communities and their variations, infer the changes in the climatic conditions that would have occurred and to deduce the depositional environment during the middle and late Holocene in the Iberá region. The profile was sampled in a ravine located in the central zone of the western margin of the Iberá System, in the locality of San Miguel. The dynamics of the vegetation and the relation of the taxa allowed establishing three pollen zones: Pollen Zone I (middle Holocene) presents a characteristic association of steppe grass. The identified
\end{abstract}

lithofacies suggests a depositional environment of waterlogged valleys, which together with the recognized vegetal palaeocommunities, would indicate humid conditions for the middle Holocene. Pollen Zones II and III (late Holocene) show an association characteristic of the herbaceous psammophyte steppe. The observed Brassicaceae increase would indicate environmental disturbances, which could reflect the combination of intense wind action under an arid climate. The identified lithofacies give evidence for an eolian environment with fluvial spills and indicate together with the recognized vegetal communities, arid to semi-arid conditions for the late Holocene.

Key words: palynology; Holocene; Iberá; reconstruction; environment; Climate; Argentina.

\section{INTRODUCCIÓN}

Con la denominación "Iberá", identificamos a uno de los humedales tropicales más importantes de la biósfera en términos de su extensión y de las especies que lo habitan, tanto animales como vegetales. Territorialmente tiene más de $12000 \mathrm{~km}^{2}$ en Argentina, aunque continúa en la República de Paraguay con el nombre de Esteros de Ñeembucú. Alcanza en total unos $45000 \mathrm{~km}^{2}$ de superficie (Neiff, 2004). Comprende un sistema hídrico complejo compuesto por esteros, bañados, lagos someros y cursos fluviales interconectados. El Macrosistema Iberá, actualmente comprendido en una zona de clima húmedo (Carnevali, 1994), es probable que haya tenido un comportamiento hipersensible a los cambios climáticos globales, reaccionando más rápidamente que las regiones francamente más áridas (González, 1987), ya que los ambientes de agua dulce son particularmente vulnerables al cambio climático debido a la naturaleza de su aislamiento y fragmentación física dentro del paisaje (Woodward et al., 2010).

Es conocido que las evidencias sobre el cambio ambiental pasado ofrecen información sobre el cambio presente y futuro, aportando datos sobre los mecanismos de variabilidad climática (Torres-Rodríguez et al., 2012). Estas variaciones en el clima en escalas temporales de centurias y de milenios modifican la distribución y la abundancia de las plantas. Por otra parte, los datos de polen fósil provenientes de secuencias sedimentarias que cuentan con cronología han sido utilizados para reconstruir la historia de la vegetación e inferir cambios en las condiciones climáticas (Watts y Bradbury, 1982). Por lo expuesto, el objetivo de este trabajo es a partir del análisis polínico y litológico de un perfil sedimentario, reconstruir las comunidades vegetales pre-

Fernandez Pacella, L., 2018, Estudio palinológico de un perfil sedimentario del Holoceno medio-tardío, oeste del Iberá, Corrientes, Argentina: Revista Mexicana de Ciencias Geológicas, v. 35, núm. 1, p. 93-101. 
dominantes y sus variaciones, inferir los cambios en las condiciones climáticas que se habrían producido y deducir el ambiente deposicional durante el Holoceno medio y tardío en la región del Iberá.

Para la República Argentina, las investigaciones palinológicas donde se reconstruyen la composición de la vegetación y sus cambios en respuesta a la variabilidad climática están centradas principalmente en el Neógeno del Noroeste (Cuadrado y Anzótegui, 1992; Mautino et al., 1997; Mautino y Anzótegui, 1998, 2002a, 2002b; Caria y Garralla, 2006; entre otros), Holoceno del Noroeste (Garralla et al., 2001, 2016; Garralla, 2002), Holoceno de la región Chaqueña (Garralla, 1998) y Holoceno de la región Pampeana (Prieto, 1989, 2016; Quattrocchio et al., 1993; 2008; Grill, 1994; Borel et al., 2001; Tonello y Prieto, 2010; Vilanova et al., 2010; Irurzun et al., 2014; Prieto et al., 2014; entre otros). Asimismo, antecedentes de estudios palinológicos sobre sedimentos cuaternarios en el nordeste de Argentina son escasos. Por lo tanto, los datos aportados en este trabajo permitirán contribuir a la reconstrucción paleoambiental de la Mesopotamia argentina.

Los resultados obtenidos se comparan con estudios realizados por otros autores en diversas regiones del país: para el Iberá (Fernandez Pacella et al., 2011), para la provincia de Entre Ríos (Tonni, 2004), para la provincia del Chaco (Iriondo, 1994), para la provincia de Santa Fe (Garralla, 1998), para el noreste de Argentina y sur de Brasil (Sandi et al., 2001), para la provincia de Buenos Aires (Tonni, 1992; Iriondo y García 1993; Quattrocchio et al., 1993, 2008; Vilanova et al., 2010; Irurzun et al., 2014), y para el Cuaternario de Argentina (Iriondo, 2010) a fin de efectuar una posible evaluación paleoclimática para el Holoceno del oeste del sistema Iberá.

\section{Características del Área}

El sistema de esteros y lagunas del Iberá, corresponde a una amplia planicie cuya somera cubeta es alimentada fundamentalmente por las lluvias (1200 a $1500 \mathrm{~mm}$ anual) y es considerado uno de los humedales más importantes de Latinoamérica. Por su extensión se halla en el decimoquinto lugar entre las planicies anegables e inundables de Sudamérica (Neiff, 1997). El Iberá ocupa el área central de la Provincia de Corrientes en forma elongada, siguiendo su eje mayor una dirección Noreste-Suroeste (Figura 1a). Constituye una compleja asociación de ambientes lénticos y lóticos desdibujados en extensas superficies de interfase.

La temperatura media anual varía entre $21^{\circ} \mathrm{C}$ al norte y $19^{\circ} \mathrm{C}$ al sur, la temperatura media del mes más frío (julio) entre $16^{\circ} \mathrm{C} \mathrm{y}$ $13{ }^{\circ} \mathrm{C}$ y la media del mes más cálido (enero) entre $27^{\circ} \mathrm{C}$ y $26^{\circ} \mathrm{C}$. Estas temperaturas estivales e invernales relativamente elevadas y su escasa variación anual definen el clima iberiano como subtropical o mesotermal (Carnevali, 1994).

Las comunidades vegetales del área de estudio se encuentran incluidas en el distrito fitogeográfico oriental de la provincia Chaqueña. El Distrito Chaqueño Oriental se encuentra sobre las planicies arenosas húmedas cubiertas por una sabana típica de Andropogon lateralis, acompañada de ciperáceas y numerosas gramíneas, desarrollándose en sectores más o menos elevados con buen drenaje; en las depresiones, este pastizal se transforma a veces en malezal ocupando superficies de lenta permeabilidad (Carnevali, 1994).

En arenas más profundas, aparecen espartillares de Elionurus muticus, ocupando suelos profundos y bien drenados de origen pluvial, al igual que pastizales de Sorghastrum agrostoides, Paspalum sp. y Spartina sp. ubicándose generalmente en suelos inundables como cañadas y bañados (Carnevali, 1994).

La pradera hidrófila de Eleocharis nodulosa, se encuentra en cuerpos de agua semi-permanetes comunes en cubetas de deflación; en esta zona, el pastizal de Andropogon lateralis se enriquece de especies graminosas y leguminosas, con la aparición de un estrato difuso

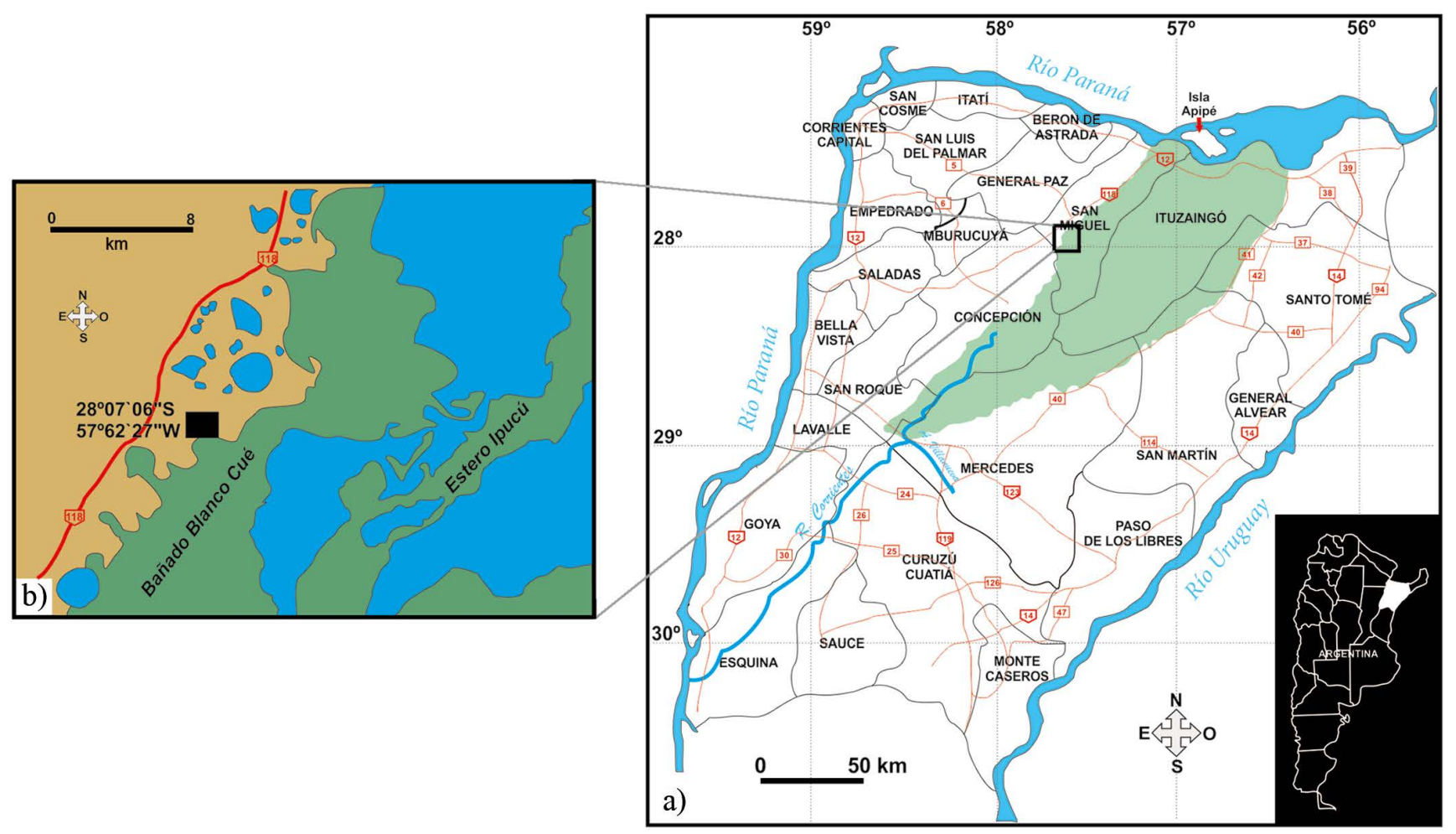

Figura 1. a) Mapa de los Esteros del Iberá en la Provincia de Corrientes y área de estudio (Extraído y modificado de Google Earth). b) Mapa de la margen oeste del Iberá indicando la ubicación geográfica del perfil fósil muestreado (extraído y modificado de Casciotta et al. 2005). 
Tabla 1. Número de muestra profundidad y edad de los sedimentos analizados.

\begin{tabular}{ccccccc}
\hline $\begin{array}{c}\mathbf{N}^{\circ} \text { de la } \\
\text { muestra }\end{array}$ & Material & $\begin{array}{c}\text { Profundidad de la } \\
\text { muestra }(\mathbf{c m})\end{array}$ & Años A.P. (C14) & $\begin{array}{c}\text { Años cal AP } \\
\text { (media ponderada) }\end{array}$ & $\begin{array}{c}\text { Rango 2 } \\
\text { Años cal A.P. }\end{array}$ & No Laboratorio \\
\hline 1 & Materia orgánica & 100 & $5620 \pm 40$ & 5968 & $5893-6043$ & 1452 (LATyR) \\
6 & Materia orgánica & 75 & $5300 \pm 70$ & 5602 & $5447-5754$ & 21482 (Beta Analytic) \\
11 & Materia orgánica & 50 & $3800 \pm 35$ & 3985 & $3951-4019$ & 21483 (Beta Analytic) \\
18 & Materia orgánica & 15 & $3200 \pm 120$ & 3486 & $3482-3492$ & 1769 (LATyR) \\
\hline
\end{tabular}

denominado "Bosque Higrófilo", extendiéndose sobre lomas de arena rojiza y "Selva Marginal o Ribereña" distribuida a manera de faja en la ribera del Paraná; se trata de una vegetación compleja alta a muy alta; también se encuentran extensos palmares de Butia yatay, así como el bosque de Schinopsis balansae y el espinillar de Prosopis affinis, que ocupan preferentemente suelos imperfectamente drenados y erosionados (Arbo y Tressens, 2002).

El perfil fue muestreado sobre una barranca ubicada en la zona central de la margen occidental del Sistema Iberá, en la localidad de San Miguel (28 $\left.07^{\prime} 06^{\prime \prime} \mathrm{S}-57^{\circ} 62^{\prime} 27^{\prime \prime} \mathrm{W}\right)$ (Figura 1b). Comprende sedimentos de origen fluvial y eólico. Según Neiff (1997), la depresión tectónica del Iberá tiene fondo irregular; si bien en el borde NO afloran algunas lomadas arenosas con su eje en sentido NE-NO, dichas lomadas constituyen relictos del modelado fluvial precedente en la misma región (Orfeo, 2005). El límite occidental del sistema es muy suave, difuso, en forma de extensos bañados y lagunas subcirculares; el fondo de esas lagunas está compuesto por arena con escaso contenido de materia orgánica (Neiff, 1997).

\section{MATERIALES Y MÉTODOS}

Del perfil sedimentario se extrajeron 20 muestras a intervalos de 5 $\mathrm{cm}$ desde la base hasta la superficie, alcanzando un espesor de $100 \mathrm{~cm}$. Las muestras obtenidas fueron procesadas en el laboratorio de palinología del Centro de Ecología Aplicada del Litoral (CECOAL) - Consejo Nacional de Investigaciones Científicas y Tecnológicas (CONICET) - Universidad Nacional del Nordeste (UNNE). Para la extracción de los granos de polen se utilizó la metodología clásica de Faegri et al. (1989) que consistió en: defloculación de arcillas con hexametafosfato de sodio al $10 \%$, eliminación de ácidos húmicos con hidróxido de sodio al 5\%, eliminación de los carbonatos con unas gotas de $\mathrm{HCl}$ al $10 \%$, separación de la materia orgánica de la inorgánica con líquidos pesados $\left(\mathrm{ZnCl}_{2}\right)$ y eliminación de los silicatos con $\mathrm{HF}$, agregándose a cada muestra dos tabletas de esporas de Lycopodium clavatum para el cálculo de la concentración polínica.

La lectura y recuento del contenido polínico se llevó a cabo de acuerdo al método propuesto por Cambón (1981). La identificación y determinación de los granos de polen se realizó mediante comparación con preparados de referencia que pertenecen a la Palinoteca de Corrientes (PAL-CTES) de la Universidad Nacional del Nordeste y bibliografía especializada (Markgraf y D’Antoni, 1978; Boelcke, 1992; Acevedo y Anzótegui, 1998; Anzótegui y Ferrucci, 1998; Cuadrado, 1998 a, b, c, d; Anzótegui, 2002; Anzótegui y Caccavari, 2002; Anzótegui y Mautino, 2002; Caccavari y Dome, 2002, 2006; Garralla y Cuadrado, 2002; Pire et al., 2006; Fernandez Pacella y Canteros, 2014; Fernandez Pacella et al., 2014).

Los datos de polen se presentan como frecuencias relativas de polen de cada taxón en un diagrama polínico elaborado mediante los programas informáticos TILIA-TILIA GRAPH y TGView 2.0.2 (Grimm, 2004). Para facilitar la lectura del diagrama se han señalado con presencias los taxones con valores menores al $1 \%$ y para los que su valor oscila entre el $1 \%$ y el $5 \%$, se ha utilizado una exageración del $5 \%$. Los taxones se han agrupado de acuerdo a sus hábitos en: arbóreos, arbustivos, herbáceos y acuáticos. Los diagramas palinológicos se dividieron en "zonas" mediante el análisis de agrupamiento en modo Q, utilizando la Distancia Euclidiana.

Las dataciones radiocarbónicas sobre materia orgánica fueron realizadas en Beta Analytic Inc. (Miami, Florida) y en el Laboratorio de Tritio y Radiocarbono (LATyR) (La Plata, Argentina) (Tabla 1). La calibración se realizó con el programa Calib Rev 7.0.4 (Stuiver y Reimer, 1993), curva de calibración del Hemisferio Sur (SHCal13, Hogg et al., 2013). En el testigo sedimentario se estimó la edad de las muestras analizadas a partir de la construcción de un modelo edad-profundidad ajustado a los fechados radiocarbónicos calibrados obtenidos (Figura 2). Se aplicó CLAM 2.2 (Blaauw, 2010), un código escrito en lenguaje R (R Core Team 2014). Para este registro sedimentario se aplicó interpolación lineal, ya que posee pocos fechados.

\section{RESULTADOS}

Sobre el perfil muestreado, se han reconocido de abajo hacia arriba: a) sedimento areno-arcilloso, compacto, con estructuras tabulares, color castaño oscuro a negro, intensamente bioturbado, b) sedimento limo-arenoso castaño claro, con estructuras entrecruzadas y tabulares,

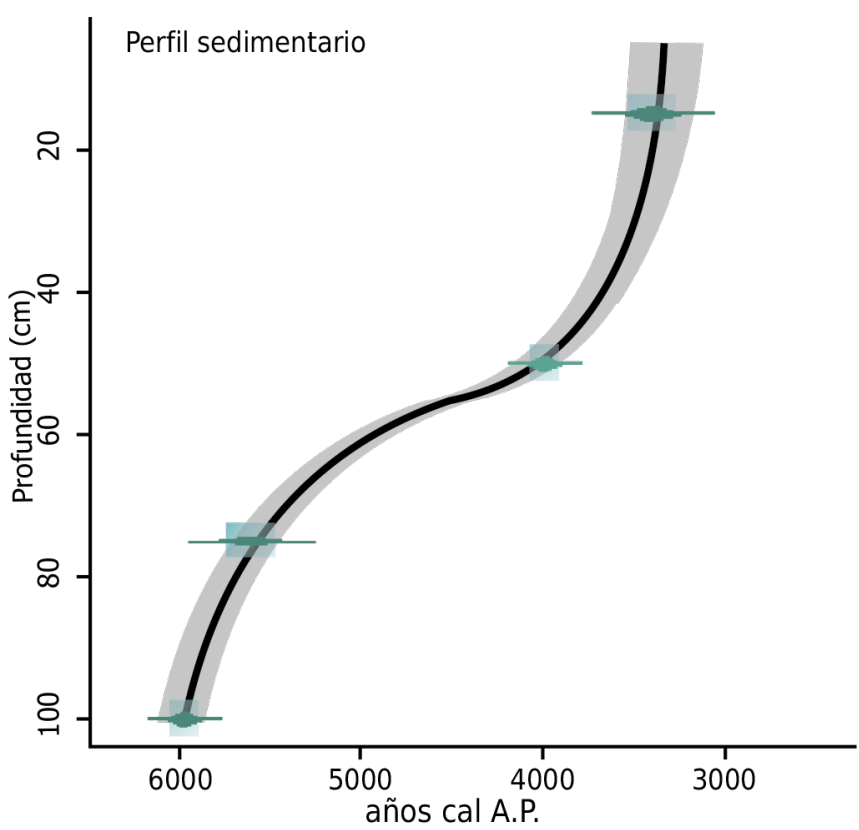

Figura 2. Modelo "edad-profundidad" ajustado a los fechados radiocarbónicos calibrados. 
compacto (Figura 3). Estos sedimentos corresponden al Holoceno medio y Holoceno tardío respectivamente, apoyado por el modelo cronológico (ver Figura 2).

Teniendo en cuenta el diagrama polínico y la representación de los granos de polen de acuerdo a la distribución en hábitos (arbóreos/ arbustivos/herbáceos/acuáticos) la sustitución entre los componentes de los distintos elementos y relación polen/condiciones ecológicas, se han podido establecer tres zonas polínicas (Figura 4):

\section{Zona I}

abarca la parte inferior del diagrama polínico $(100-65 \mathrm{~cm})$. Según el modelo cronológico y los niveles fechados corresponde al Holoceno medio, alrededor de 5968 y 5000 años cal A.P. (ver Figura 2). Desde el punto de vista litológico corresponde a sedimentos areno-arcillosos. Está caracterizada por Poaceae, Asteraceae, ChenopodiaceaeAmaranthaceae. Poaceae, con un registro continuo en todo el perfil, alcanza en esta zona su máximo porcentaje, variando entre $23 \%$ y $58 \%$, lo mismo sucede con Asteraceae entre $18 \%$ y $33 \%$, acompañadas por Chenopodiaceae-Amaranthaceae, variando entre 5\% y $20 \%$. La familia Apiaceae oscila entre 5\% y $10 \%$. El monte arbustivo y arbóreo está pobremente representado por Anacardiaceae (Schinus sp.) y Ephedraceae (Ephedra sp.). El registro de polen de larga distancia (Myrtaceae y Alnus sp.) es escaso, no superando el 5\% en ambos casos. La vegetación acuática y palustre varía entre $2 \%$ y $8 \%$. La concentración polínica total disminuye de 24370 granos/gramo en los niveles basales de la zona a 16849 granos/gramo en los niveles superiores. El grupo más representativo en toda la zona corresponde a Poaceae con 10858 granos/gramo (Figura 5).

\section{Zona II}

Ubicada en la parte media del diagrama polínico $(60$ - $30 \mathrm{~cm})$. Según el modelo cronológico y los niveles fechados corresponde al Holoceno tardío, alrededor de 4900 y 3300 años cal A.P. (ver Figura 2). Desde el punto de vista litológico corresponde a sedimentos limo-arenosos. Está identificada por la asociación de Chenopodiaceae-Amaranthaceae,

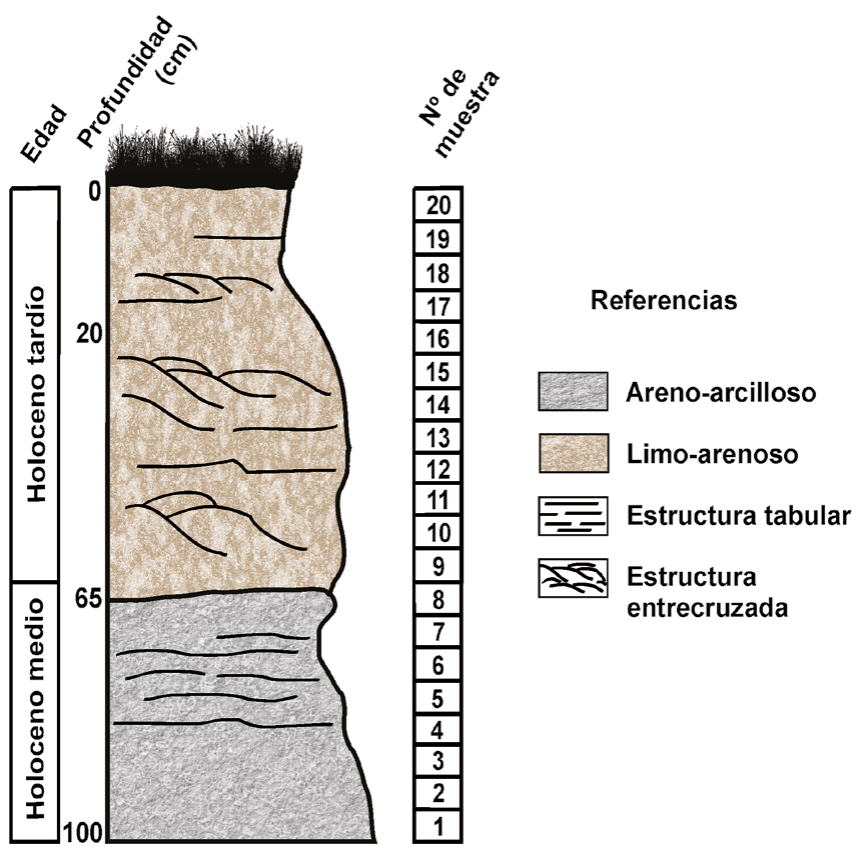

Figura 3. Esquema estratigráfico del perfil fósil muestreado. Incluye profundidad, edad, y número de muestra.
Brassicaceae, Asteraceae y Poaceae. Con respecto a la zona anterior, se registra un aumento de las Chenopodiaceae-Amaranthaceae que varían entre $18 \%$ y $40 \%$, junto con Brassicaceae que alcanzan un valor máximo de 36\%. Se observa una disminución de Asteraceae que oscila entre $11 \%$ y $27 \%$ y de Poaceae que varía entre $9 \%$ y $27 \%$. Entre los taxones propios de la estepa herbácea psamófila se encuentran: la familia Apiaceae (10\%), Caryophyllaceae, Scrophulariaceae, Polygonaceae (Rumex sp.) y Urticaceae con valores inferiores al 5\%. Como representantes del monte arbustivo y arbóreo se distinguen: Ephedraceae (Ephedra sp.), Anacardiaceae (Schinus sp.) y Fabaceae (Prosopis sp. y Acacia sp.) con valores que no superan el 5\%. La presencia del polen extra-regional (Myrtaceae y Alnus sp.) es escasa, no superando el $5 \%$, al igual que la vegetación acuática y palustre. Los máximos valores de concentración polínica varían desde 29056 hasta 23807 granos/gramo destacándose las Chenopodiaceae-Amaranthaceae con 7488 granos/gramo y Brassicaceae con 6739 granos/gramo (Figura 5).

\section{Zona III}

Definida como la parte superior del diagrama polínico $(25-5 \mathrm{~cm})$. Según el modelo cronológico y los niveles fechados corresponde al Holoceno tardío, a partir de 3300 años cal A.P. en adelante (ver Figura 2). Desde el punto de vista litológico corresponde a sedimentos limoarenosos. Está identificada por Chenopodiaceae-Amaranthaceae, Asteraceae y Poaceae. Con respecto a la zona anterior, se registra un aumento en las familias Chenopodiaceae-Amaranthaceae que varían entre $23 \%$ y $65 \%$, y por otra parte, una notable disminución en las Asteraceae que oscilan entre $10 \%$ y $16 \%$, en las Brassicaceae (20\%) y en las Poaceae (5\% y $11 \%)$. El monte arbustivo y arbóreo está representado por Fabaceae (Prosopis sp. 8\% y Acacia sp. 22\%), Ephedraceae (Ephedra sp.) y Anacardiaceae (Schinus sp.), con valores inferiores al 5\%. La presencia de la vegetación acuática y palustre es casi nula. Esta zona alcanza los máximos valores de concentración polínica variando entre 29244 y 18371 granos/gramo. También se destacan las familias Chenopodiaceae-Amaranthaceae con 11607 granos/gramo y Brassicaceae con 8798 granos/gramo (Figura 5).

\section{DISCUSIÓN}

Del análisis de las asociaciones polínicas recuperadas se reconstruyen las paleocomunidades vegetales. Además, al relacionar los datos de vegetación y de las litofacies correspondientes, pueden inferirse las condiciones paleoclimáticas que habrían imperado en el área de estudio (Tabla 2).

El Holoceno medio (entre 5968 - 5000 años cal A.P.) caracterizado por Poaceae, Asteraceae, Chenopodiaceae-Amaranthaceae presenta una asociación característica de la estepa graminosa frecuente en ambientes húmedos.

Las litofacies identificadas sugieren un ambiente depositacional de valles anegadizos. Isla (1989) infiere que durante el Holoceno temprano y medio, las condiciones climáticas fueron subhúmedashúmedas, y el nivel del mar alcanzó su nivel moderno alrededor de los 8000 años A.P., y aumentó 2.2 a $2.5 \mathrm{~m}$ con respecto al nivel actual aproximadamente a los 6000 años A.P. Esto se debió a que el Holoceno medio se encontraba caracterizado por condiciones climáticas marcadas de máxima insolación, es decir que la principal forzante estaba relacionada a los parámetros orbitales, en consecuencia se produjo la disminución del tamaño de los glaciares, el aumento del nivel del mar y la concentración de gases de efecto invernadero (Apaéstegui et al., 2014). Un debilitamiento de la surgencia de aguas frías en las costas, ascenso de la capa de inversión térmica, disminución de la nubosidad y vientos de debilitados, parecen concordantes con la transgresión 


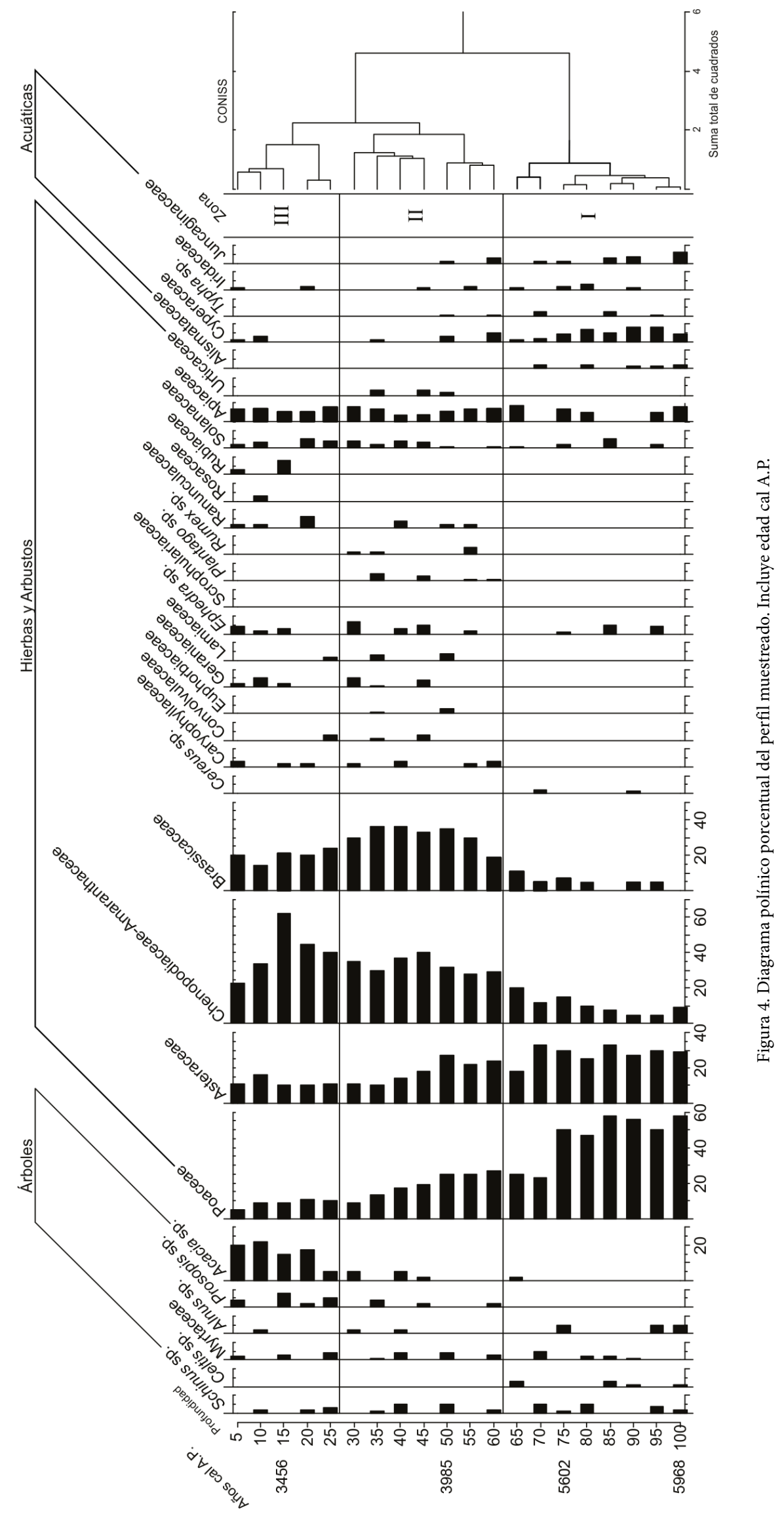




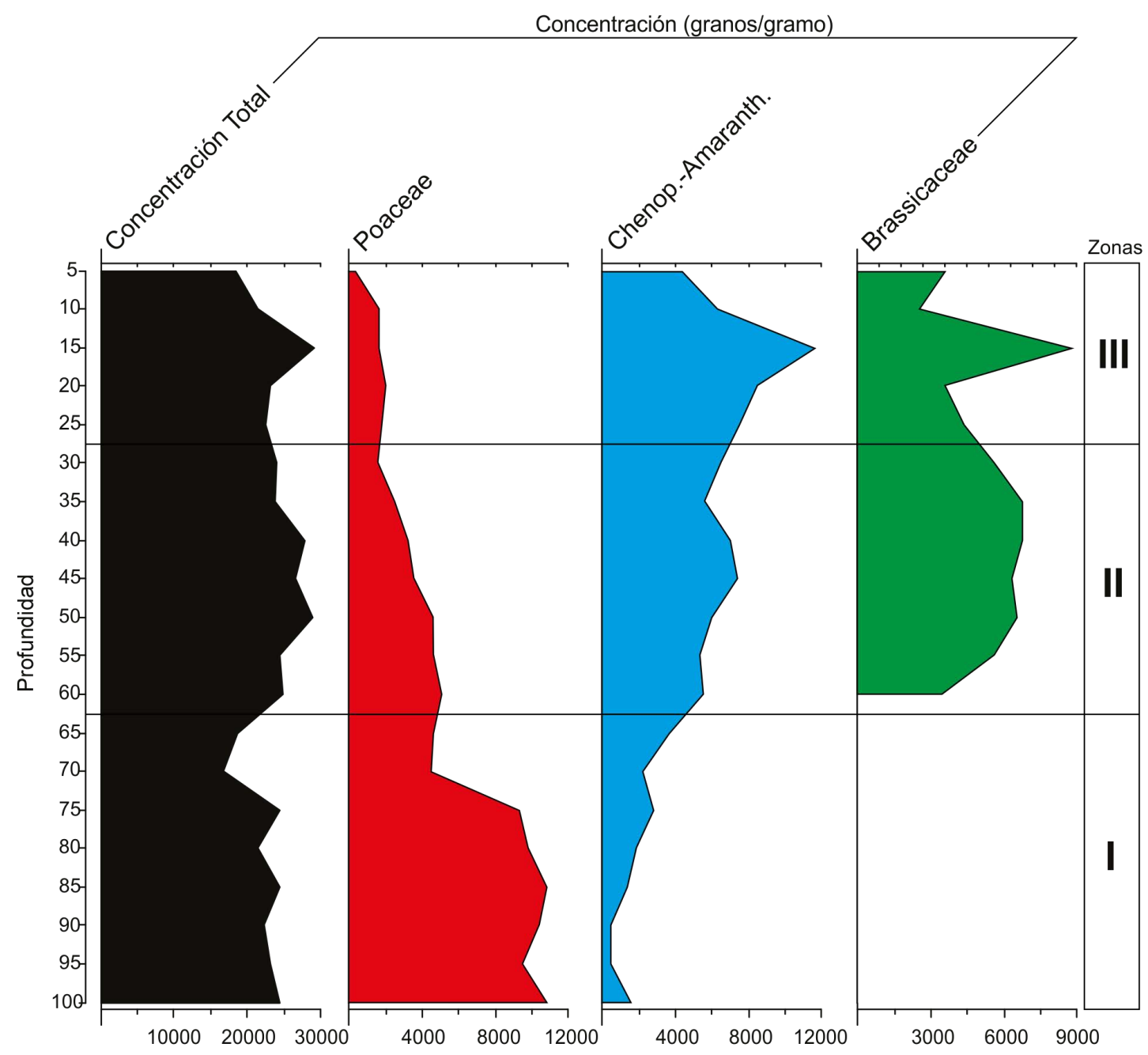

Figura 5. Diagrama de concentración total (granos/gramo) y de los grupos más representativos.

marina documentada entre 6000 - 5000 años A.P., evento relacionado probablemente con el máximo de temperaturas del Holoceno medio (Villa-Martinez y Villagrán, 1997).

El Holoceno medio estaría representado por una asociación propia de la estepa graminosa y se correlacionaría con un episodio de desbordamiento, señalando condiciones ambientales húmedas. Este episodio de desbordamiento podría deberse a la transgresión marina ocurrida durante el Holoceno medio que habría inundado los lechos de los ríos. Sandi et al. (2001) indican, del análisis de cuatro pozos para el noreste de Argentina y sur del Brasil (en zonas aledañas al río Paraná en su curso medio y superior), un alto porcentaje de algas evidenciando un incremento en el nivel ácueo que podría interpretarse como un pulso de inundación ocurrido durante el Holoceno medio. Las condiciones ambientales descritas concuerdan con lo publicado por Fernandez Pacella et al. (2011) quienes demuestran para el noroeste del Iberá, y entre 6284 - 5241 años cal A.P., el predominio de la vegetación herbácea de ambientes húmedos, coincidiendo con lo publicado por Iriondo (1994) señalando que, durante el Holoceno temprano y medio en el norte de Argentina y principalmente en áreas próximas al río Paraná (en la provincia del Chaco), la acción de la erosión retrogradante formó redes fluviales, que se sabe estaban bien desarrolladas por la presencia de sedimentos fluviales. Por otra parte, Quattrocchio et al. (2008) mencionan en base a depósitos continentales de dinocistos y acritarcos de la parte suroccidental de la provincia de Buenos Aires, que la temperatura y la humedad alcanzaron su máximo durante el Holoceno medio. Igualmente, Vilanova et al. (2010) establecen para la provincia de Buenos Aires, entre 6500 años A.P. y hasta los 3700 años A.P., condiciones subhúmedas con el reemplazo de vegetación halófita por comunidades de humedales, en respuesta a una elevación del nivel de agua.

El Holoceno tardío (a partir de 4900 años cal A.P. en adelante) caracterizado por Chenopodiaceae-Amaranthaceae, Brassicaceae, Asteraceae y Poaceae presenta una asociación característica de estepa herbácea psamófila. El aumento observado en las Brassicaceae, estaría indicando disturbios ambientales, los cuales podrían reflejar la combinación de una intensa acción eólica bajo un clima árido (León y Anderson, 1983).

Las litofacies identificadas señalan un ambiente eólico con derrames fluviales y junto a las comunidades vegetales reconocidas, indicarían para el Holoceno tardío condiciones áridas a semiáridas.

Las condiciones ambientales descritas para este período concuerdan con lo publicado por Garralla (1998), quien señala ambientes similares alrededor de los 3500 años A.P. para el Norte de la provincia de Santa Fe (al O del presente sitio de estudio), indicando el predominio de vegetación de bosque xerohalófilo y el desarrollo de períodos climáticos secos, al igual que Iriondo (2010), quien destaca la presencia, al sur del 
Tabla 2. Paleoambientes y paleoclimas de la zona estudiada de acuerdo a la edad y el origen de los sedimentos.

\begin{tabular}{|c|c|c|c|c|c|c|}
\hline Edad & $\begin{array}{l}\text { Origen del } \\
\text { sedimento }\end{array}$ & & Zonas polínicas & $\begin{array}{l}\text { Comunidades } \\
\text { vegetales }\end{array}$ & Paleo-ambiente & Paleo-clima \\
\hline $\begin{array}{l}\text { Holoceno } \\
\text { tardío }\end{array}$ & eólico & $\begin{array}{l}\text { III } \\
\text { II }\end{array}$ & $\begin{array}{l}\text { Chenopodiaceae } \\
\text { Amaranthaceae } \\
\text { Brassicaceae } \\
\text { Asteraceae } \\
\text { Poaceae }\end{array}$ & $\begin{array}{l}\text { estepa } \\
\text { herbácea } \\
\text { psamófila }\end{array}$ & $\begin{array}{l}\text { eólico con } \\
\text { derrames } \\
\text { fluviales }\end{array}$ & $\begin{array}{l}\text { árido a } \\
\text { semiárido }\end{array}$ \\
\hline $\begin{array}{l}\text { Holoceno } \\
\text { medio }\end{array}$ & fluvial & I & $\begin{array}{l}\text { Poaceae } \\
\text { Asteraceae } \\
\text { Chenopodiaceae } \\
\text { Amaranthaceae }\end{array}$ & $\begin{array}{l}\text { estepa } \\
\text { graminosa }\end{array}$ & $\begin{array}{l}\text { valles } \\
\text { anegadizos }\end{array}$ & $\begin{array}{l}\text { subhúmedo a } \\
\text { húmedo }\end{array}$ \\
\hline
\end{tabular}

sistema Iberá, campos de dunas eólicas generadas durante las etapas secas del Holoceno tardío. Además, Iriondo y García (1993) mencionan para la llanura chacopampeana clima semiárido para el intervalo 3500 y 1000 años A.P., con intensa actividad eólica. En un trabajo posterior (Iriondo, 1999), acota este período seco entre 3500 y 1400 años. Por otro lado, Vilanova et al. (2010) establecen para la provincia de Buenos Aires, alrededor de los 3400 años A.P. la deposición de sedimentos esporádicos y una tendencia hacia la desecación de humedales en un estado medio climático subhúmedo a seco. Asimismo, Quattrocchio et al. (2008), indican para depósitos continentales de la parte suroccidental de la provincia de Buenos Aires, alrededor de los 3000 años A.P., el desarrollo de estepa herbácea psamófila, sugiriendo condiciones áridas a semiáridas. Además, Tonni (1992) indica condiciones áridas a semiáridas, en base a mamíferos fósiles, en el Holoceno tardío anterior a los 1000 años A.P., para las provincias de Buenos Aires y Entre Ríos. Igualmente, Tonni (2004), apoya la hipótesis de que el período árido que parece haber comenzado hace alrededor de 3500 años (Iriondo, 1999), se extendió en el sudoeste de la provincia de Corrientes, hasta por lo menos el lapso de 950 a 1200 años A.P.

En América del Sur, el Holoceno temprano y medio se caracterizaron por un cambio natural del clima debido a las variaciones en los parámetros orbitales (la excentricidad de la órbita terrestre, la inclinación de la Tierra, o el movimiento del su eje de rotación) (Apaéstegui et al., 2014). La variación de estos parámetros orbitales modificaron constantemente la posición y la exposición de la Tierra al Sol, generando una fuerte variabilidad climática, provocando la alternancia de períodos de inundación y/o períodos de alta evaporación bajo condiciones climáticas subhúmedas (Apaéstegui et al., 2014). A partir del Holoceno tardío, como resultado de la interacción de las masas de aire marítimas tropicales y marítimas polares tal como sucede en la actualidad, se registrarían condiciones frías y húmedas en el sur de Argentina (Tierra del Fuego, Heusser, 1989) y áridas a semiáridas en el centro y norte de Argentina (Quattrocchio et al., 1993). Durante los últimos 3000 años, América del Sur ha pasado por cambios en los patrones de distribución de las precipitaciones durante la Anomalía Climática Medieval, donde la variabilidad del clima se caracterizó por una disminución de las precipitaciones en el Amazona occidental, incluyendo los Andes (Apaéstegui et al., 2014).

\section{CONCLUSIÓN}

Los estudios palinológicos aquí efectuados permiten sugerir que durante la evolución de los depósitos sedimentarios ocurridos en el Holoceno medio y tardío, en los Esteros de Iberá ocurrieron diversos cambios en la vegetación, como consecuencia de las variaciones ambientales. Dichas variaciones consistieron en la sucesión de episodios húmedos a secos en la región. Desde el punto de vista fisonómico, se observa el dominio de las formaciones herbáceas, mientras que las leñosas son de escasa significancia.

Las características más relevantes de los estudios cuaternarios del oeste de los Esteros del Iberá expuestos anteriormente llevan a las siguientes conclusiones: durante el Holoceno medio habrían predominado condiciones ambientales húmedas correlacionadas con episodios de desbordamientos, debido posiblemente al aumento relativo del nivel del mar que inundó los lechos de los ríos y facilitó la propagación de una estepa graminosa. Durante el Holoceno tardío las condiciones ambientales debieron ser más áridas, puesto que la región se encontraría caracterizada por la estepa psamófila y relacionada con una intensa acción eólica, la cual se observa con el aumento de las Brassicaceae; ya que estas indican disturbios ambientales.

Aún quedan amplias áreas con excelentes afloramientos, que son potenciales para el análisis palinológico y permitirán ampliar la información regional sobre la vegetación del pasado. Basado en la movilidad del río Paraná como agente modelador del paisaje, la realización de este trabajo permitirá conocer, a través del análisis polínico en el área de estudio, la paleovegetación del Macrosistema Iberá e inferir su evolución. A su vez, aportará información para discriminar sub-ambientes y establecer cambios paleoambientales, paleoclimáticos y permitir la reconstrucción paleoecológica del Holoceno de la región.

\section{REFERENCIAS}

Acevedo, T.L., Anzótegui, L.M., 1998, Myrtaceae, en Pire, S.M., Anzótegui, L.M., Cuadrado, G.A. (eds.), Flora Polínica del Nordeste argentino Vol. I: Corrientes, Argentina, EUDENE, 67-80.

Anzótegui, L.M., 2002, Anacardiaceae, en Pire, S.M., Anzótegui, L.M., Cuadrado, G.A. (eds.), Flora Polínica del Nordeste argentino Vol. II: Corrientes, Argentina, EUDENE, 19-26.

Anzótegui, L.M., Ferrucci, M.S., 1998, Sapindaceae, en Pire, S.M., Anzótegui, L.M., Cuadrado, G.A. (eds.), Flora Polínica del Nordeste argentino Vol. I: Corrientes, Argentina, EUDENE, 95-110.

Anzótegui, L.M., Caccavari, M.A., 2002, Vitaceae, en Pire, S.M., Anzótegui, L.M., Cuadrado, G.A. (eds.), Flora Polínica del Nordeste argentino Vol. II: Corrientes, Argentina, EUDENE, 141-147.

Anzótegui, L.M., Mautino, L.R., 2002, Celtidaceae, en Pire, S.M., Anzótegui, L.M., Cuadrado, G.A. (eds.), Flora Polínica del Nordeste argentino Vol. II: Corrientes, Argentina, EUDENE, 61-64.

Apaestegui, J., Sifeddine, A., Turcq, B., da Cruz, W., Guyot, J.L., Cheng, H., Bernal, J.P., Leite da Silva Dias, P., Albuquerque, A.L.S., Cordeiro, R.C., Belem, A., Moreira-Turcq, P., Gutierrez, D., Ortlieb, L., Velazco, F., 2014, Cambios Climáticos del Holoceno, en Gonzalez Molina, S., Vacher, J. (coord.), El Perú frente al cambio climático: Resultados de investigaciones franco-peruanos: Perú, IRD, 33-45 pp.

Arbo, M., Tressens, S., 2002, Flora del Iberá: Corrientes, Argentina, EUDENE, 613 pp. 
Blaauw, M., 2010, Methods and code for "classical" age-modelling of radiocarbon sequences: Quaternary Geochronology, 5, 512-518.

Boelcke, O., 1992, Plantas vasculares de la Argentina nativas y exóticas: Buenos Aires, Hemisferio Sur S.A., 334 pp.

Borel, C.M., Bianchinotti, M.V., Quattrocchio, M.E., 2001, Palinomorfos fúngicos del Pleistoceno-Holoceno en el valle del Arroyo Chasicó, provincia de Buenos Aires, Argentina: Polen, 11, 21-37.

Caccavari, M.A., Dome, E., 2002, Fabaceae - Mimosoideae. Tribu: Acacieae, en Pire, S.M., Anzótegui, L.M., Cuadrado, G.A. (eds.), Flora Polínica del Nordeste argentino Vol. II: Corrientes, Argentina, EUDENE, 65-72.

Caccavari, M.A., Dome, E., 2006, Fabaceae - Mimosoideae. Tribu: Mimoseae, en Pire, S.M., Anzótegui, L.M., Cuadrado, G.A. (eds.), Flora Polínica del Nordeste argentino Vol. III: Corrientes, Argentina, EUDENE, 55-69.

Cambón, G., 1981, Relations entre le contenu pollinique de l'atmosphere et le couvert vegetal mediterrannee occidentale a Montpellier (France), Valencia (Espagne) et Oran (Algerie): Montpellier, Francia, Université des Sciences et Techniques du Languedoc, Tesis Doctoral, $105 \mathrm{pp}$.

Caria, M., Garrala, S.S., 2006, Evolución Paleoambiental del Sitio Acequia (Trancas-Tucumán-Argentina) a partir de indicadores polínicos (resumen), en III Congreso Argentino de Cuaternario y Geomorfología, Tucumán: Tucumán, Argentina, Actas de trabajos, 75-84.

Carnevali, R., 1994, Fitogeografía de la Provincia de Corrientes: Corrientes, Argentina, Gobierno de la Provincia de Corrientes - Instituto Nacional de Tecnología Agropecuaria, 324 pp.

Casciotta, J., Almirón, A., Bechara, J, 2005, Peces del Iberá: hábitat y diversidad: La Plata, Argentina, Grafikar, 244 pp.

Cuadrado, G.A., 1998a, Amaranthaceae, en Pire, S.M., Anzótegui, L.M. Cuadrado, G.A. (eds.), Flora Polínica del Nordeste argentino Vol. I: Corrientes, Argentina, EUDENE, 23-33.

Cuadrado, G.A., 1998b, Chenopodiaceae, en Pire, S.M., Anzótegui, L.M., Cuadrado, G.A. (eds.), Flora Polínica del Nordeste argentino Vol. I: Corrientes, Argentina, EUDENE, 59-65.

Cuadrado, G.A., 1998c, Polygalaceae, en Pire, S.M., Anzótegui, L.M., Cuadrado, G.A. (eds.), Flora Polínica del Nordeste argentino Vol. I: Corrientes, Argentina, EUDENE, 81-88.

Cuadrado, G.A., 1998d, Sapotaceae, en Pire, S.M., Anzótegui, L.M., Cuadrado, G.A. (eds.), Flora Polínica del Nordeste argentino Vol. I: Corrientes, Argentina, EUDENE, 111-117.

Cuadrado, A.G., Anzótegui, L.M., 1992, Resultados preliminares del análisis palinológico de la Formación Palo Pintado, Mioceno de Salta, Argentina: Asociación Paleontológica Argentina Publicación Especial, 2, 51-53.

Faegri, K., Kaland, P.E., Krzywinski, K.J., 1989, Textbook of Pollen Analysis: New York, John Wiley and Sons, 328 pp.

Fernández Pacella L., Garralla S.S., Anzótegui L.M., 2011, Cambios de la vegetación durante el Holoceno en la región Norte del Iberá. Provincia de Corrientes, Argentina: Revista de Biología Tropical de Costa Rica, 59(1), 103-112

Fernández Pacella, L., Canteros, O., 2014, Poaceae. Tribus: Andropogoneae, Bromeae, Cynodonteae, Eragrostidae, Olyreae y Oryzeae, en Pire, S.M. Anzótegui, L.M., Cuadrado, G.A. (eds.), Flora Polínica del Nordeste argentino Vol. IV: Corrientes, Argentina, EUDENE, 119-126.

Fernández Pacella L., Anzótegui L.M., Mautino L.R., 2014, Fabaceae Mimosoideae. Tribu: Mimoseae-Prosopis, en Pire, S.M., Anzótegui, L.M., Cuadrado, G. A. (eds.), Flora Polínica del Nordeste argentino Vol. IV: Corrientes, Argentina, EUDENE, 88-94.

Garralla, S.S., 1998, Estudio palinológico de una secuencia sedimentaria del Holoceno, Norte de Santa Fe, Argentina: Polen, 9, 17-27.

Garralla, S.S., 2002, Análisis polínico de una secuencia sedimentaria del Holoceno Tardío en el Abra del Infiernillo, Tucumán, Argentina: Polen, 12, 53-63.

Garralla, S.S., Cuadrado, G.A., 2002, Meliaceae, en Pire, S.M., Anzótegui, L.M., Cuadrado, G. A. (eds.), Flora Polínica del Nordeste argentino Vol. II: Corrientes, Argentina, EUDENE, 95-100.

Garralla, S.S., Muruaga, C., Herbst, R., 2001, Lago El Ricón, Holoceno del departamento de Tafí del Valle, provincia de Tucumán (Argentina): palinología y facies sedimentarias: Asociación Paleontológica Argentina Publicación Especial, 91-99.

Garralla S.S., Anzótegui L.M., Mautino L.R., 2016, Relaciones Paleofloristicas del Mioceno-Plioceno del Norte Argentino. Publicación Electrónica de la Asociación Paleontológica Argentina (PE-APA), vol. 16, Buenos Aires. González M., 1987, El paleoclima del Cuaternario en el territorio de la República Argentina: Revista Asociación Geológica Argentina, 42(3), 231-243.

Grill, S., 1994, Estratigrafía y paleoambientes del Cuaternario en base a palinomorfos, en la cuenca del arroyo Napostá Grande, Provincia de Buenos Aires: Bahía Blanca, Argentina, Universidad Nacional del Sur, Tesis Doctoral $145 \mathrm{pp}$.

Grimm, E., 2004, TGView 2.0.2: Springfield, Illinois State Museum, Research and Collection Center.

Heusser, C., 1989, Late Quaternary vegetation and climate of southern Tierra del Fuego: Quaternary Research, 31, 396-406.

Hogg, A.G., Hua, Q. Blackwell, P.G., Niu, M., Buck, C.E., Guilderson, T.P., Heaton, T.J., Palmer, J.G., Reimer, P.J., Reimer, R.W., Turney, C.S.M, y Zimmerman, S.R.J., 2013, SHCal13 southern hemisphere calibration, 0 - 50,000 cal BP: Radiocarbon, 55, 1889-1903.

Iriondo, M.H., 1994, Los climas cuaternarios de la región Pampeana: Comunicaciones del Museo Provincial de Ciencias Naturales "Florentino Ameghino" (N. S), 4(2), 1-48.

Iriondo, M.H., 1999, Climatic changes in the South American plains: records of a continent-scale oscillation: Quaternary International, 57/58, 93-112.

Iriondo, M.H., 2010, Geología del Cuaternario en Argentina: Corrientes, Argentina, Editorial Moglia, 100 pp.

Iriondo. M.H., García, N., 1993, Climatic variations in the Argentine plains during the last 18,000 years: Palaeogeography, Palaeoclimatology, Palaeoecology, 101, 209-220.

Irurzun, M.A, Gogorza, C.S., Sinito, A.M., Chaparro, M.A., Prieto, A.R., Laprida, C., Lirio, J.M., Navas, A., Nuñez, H., 2014, A high resolution paleoclimate record for the last $4800 \mathrm{cal}$. years B.P. on Lake La Brava SE Pampa plains, Argentina: Geofísica Internacional, 53, 365-383.

Isla, F.I., 1989, Holocene sea-level fluctuation in the southern Hemisphere: Quaternary Science Reviews, 8, 359-368.

León, R., Anderson. D., 1983, El límite occidental del pastizal pampeano: Tuexenia, 3, 67-82

Markgraf, V., D`Antoni, H.L., 1978, Polen Flora of Argentina - Modern Spore and Pollen Types of Pteridophyta, Gymnospermae and Angiospermae: Tucson Arizona, The University of Arizona Press., 208 pp.

Mautino, L.R., Anzótegui, L.M., Herbst, R., 1997, Análisis palinológico de la localidad Nacimientos de Abajo, en la sierra de Hualfin, Departamento Belén, Catamarca, Argentina: Geociencias, 2, 121-127.

Mautino, L.R., Anzótegui L.M., 1998, Palinología de la Formación Chiquimil (Mioceno superior) Localidad Vallecito, Provincia de Catamarca. Parte I: Especies Nuevas, Ameghiniana, 35(2), 227-233.

Mautino, L.R., Anzótegui L.M., 2002a, Palinología de la Formación Chiquimil (Mioceno superior), Vallecito, provincia de Catamarca. Parte 2. Polen: Ameghiniana, 39 (3), 257-270.

Mautino, L.R., Anzótegui, L.M., 2002b, Palinología de la Formación Chiquimil (Mioceno superior) Vallecito, provincia de Catamarca. Parte 3. Polen: Ameghiniana, 39(3), 271-284.

Neiff J.J., 1997, Ecología evolutiva del macrosistema Iberá (Corrientes, Argentina): Santa Fe, Argentina, Universidad Nacional del Litoral, Tesis de Maestría, 138 pp.

Neiff, J.J., 2004, El Iberá... ¿ en peligro?: Buenos Aires, Ed. Fundación Vida Silvestre, Argentina, $89 \mathrm{pp}$.

Orfeo, O., 2005, Historia Geológica del Iberá, provincia de Corrientes, como escenario de biodiversidad: Miscelánea INSUGEO, 14, 71-78.

Pire, S.M., Anzótegui, L.M., Mautino, L.R., Garralla, S.S., 2006, Acanthaceae, en Pire, S.M., Anzótegui, L.M., Cuadrado, G.A. (eds.), Flora Polínica del Nordeste argentino Vol. III: Corrientes, Argentina, EUDENE, 15-38.

Prieto, A.R., 1989, Palinología de Empalme Querandíes, Provincia de Buenos Aires. Un modelo paleoambiental para el Pleistoceno tardío - Holoceno: Mar del Plata, Argentina, Universidad Nacional de Mar del Plata, Tesis Doctoral, $207 \mathrm{pp}$.

Prieto, A.R., 2016, Análisis e Interpretación de Registros Palinológicos de Sucesiones Aluviales de la Región Pampeana: Revisión e Implicancias para la Reconstrucción de la Vegetación y el Clima durante el Pleistoceno Tardio - Holoceno: Publicación Electrónica de la Asociación Paleontológica Argentina, 16, 148-167.

Prieto, A.R., Romero, M.V., Vilanova, I., Bettis III, A.E., Espinosa, M.A., Haj, A.E., Gómez, L., Bruno, L.I., 2014, A multi-proxy study of Holocene 
environmental change recorded in alluvial deposits along the southern coast of the Pampa region, Argentina: Journal of Quaternary Science, $29,329-342$.

Quattrocchio, M., Deschamps, C., Zavala, C., Borromei, A., Grill, S., Guerstein, R., 1993, Cuaternario del sur de la provincia de Buenos Aires. Estratigrafía e inferencias paleoambientales, en Iriondo, M. (ed.), El Holoceno en Argentina: Paraná, Cadinqua, 2, 22-34.

Quattrocchio, M., Borromei, A.M., Deschamps, C., Grill, S., 2008, Landscape evolution and climate changes in the late Pleistocene - Holocene, southern pampa (Argentina): evidence from palynology, mammals and sedimentology: Quaternary International, 123, 138-181.

R Core Team, 2014, R: A language and environment for statistical computing. R Foundation for Statistical Computing, Vienna, Austria, <http://www.Rproject.org/>, consultado el 6 de febrero de 2018.

Sandi, A.A., Anzótegui, L.M., Garralla, S.S., 2001, Estudio palinológico del Holoceno en la planicie de inundación del río Paraná Superior, Estado de Paraná, Brasil (resumen), en X Reuniao de Paleobotánicos e Palinologos, Guarulhos, Sao Paulo: Sao Paulo, Geociencias, Actas, 5, 260.

Stuiver, M., Reimer, P., 1993, Extended 14C database and revised CALIB radiocarbon calibration program: Radiocarbon, 35, 215-230.

Tonello, M.S., Prieto, A.R., 2010, Tendencias climáticas para los pastizales pampeanos durante el Pleistoceno tardío-Holoceno: estimaciones cuantitativas basadas en secuencias polínicas fósiles: Ameghiniana, 7(4), 501-514.

Tonni, E., 1992, Los mamíferos y el clima del Holoceno en la provincia de Buenos Aires, en Iriondo, M. (ed.), El Holoceno en Argentina: Paraná, Cadinqua, 1, 64-78.
Tonni, E., 2004, Faunas y Clima en el Cuaternario de la Mesopotamia Argentina, en Aceñolaza, F. (Coord.), Temas de la Biodiversidad del Litoral fluvial argentino: Buenos Aires, INSUGEO, 12, 31-38.

Torres-Rodríguez, E., Lozano-García, S., Figueroa-Rangel, B.L., OrtegaGuerrero, B., Vázquez-Castro, G., 2012. Cambio ambiental y respuestas de la vegetación de los últimos 17,000 años en el centro de México: el registro del lago de Zirahuén: Revista Mexicana de Ciencias Geológicas, 29(3), 764-778.

Vilanova, I., Prieto, A.R., Stutz, S., Bettis III, A., 2010, Holocene vegetation changes along the southeastern coast of the Argentinean Pampa: Palaeogeography Palaeoclimatology Palaeoecology, 298, 210-223.

Villa-Martinez, R., Villagrán, C., 1997, Historia de la vegetación de bosques pantanosos de la costa de Chile central durante el Holoceno Medio y Tardío: Revista Chilena de Historia Natural, 70, 391-401.

Watts, W.A., Bradbury, J.P., 1982, Paleoecological Studies at lake Patzcuaro on the West-central Mexican Plateau and at Chalco in the Basin of Mexico: Quaternary Research, 17, 56-20.

Woodward, G., Perkins, D.M., Brown, L.E., 2010, Climate change and freshwater ecosystems: impacts across multiple levels of organization: Philosophical Transactions of the Royal Society, 365, 2093-2106.

Manuscrito recibido: junio 26, 2017

Manuscrito corregido recibido: marzo 6, 2018

Manuscrito aceptado: marzo 7, 2018 